\title{
A quasar under a microlensing caustic
}

\section{Timo Anguita*, Robert Schmidt}

Zentrum für Astronomie der Universität Heidelberg

E-mail: tanguita@ari.uni-heidelberg.de

Using observations of the high magnification event seen in image C of quasar Q2237+0305 during 1999 we have obtained constraints on the structure of the quasar's central engine. Data taken with the Apache Point Observatory $3.5 \mathrm{mts}$ telescope in the g' and the r' bands shows a smaller amplitude of the event in the r' filter. We compare these observations and those presented by the OGLE team with synthetic source plane microlensing magnification patterns in order to obtain constraints on the size of the accretion disk regions contributing to each band. We obtain for the Gaussian width $1.20 \times 10^{15} \sqrt{M / 0.1 M_{\odot}} \mathrm{cm} \lesssim \sigma_{g^{\prime}} \lesssim 7.96 \times 10^{15} \sqrt{M / 0.1 M_{\odot}} \mathrm{cm}$, where $\mathrm{M}$ is the mean microlensing mass, and a ratio $\sigma_{r^{\prime}} / \sigma_{g^{\prime}}=1.25_{-0.15}^{+0.45}$. With the limits on the velocity of the lensing galaxy from Gil-Merino et al. (2005)[2] as our only prior, we obtain $0.60 \times 10^{15} \sqrt{M / 0.1 M_{\odot}} \mathrm{cm} \lesssim \sigma_{g^{\prime}} \lesssim 1.57 \times 10^{15} \sqrt{M / 0.1 M_{\odot}} \mathrm{cm}$ and a ratio $\sigma_{r^{\prime}} / \sigma_{g^{\prime}}=1.45_{-0.25}^{+0.90}$ (all values at 68 percent confidence). Additionally, from our microlensing simulations we find that, during the chromatic microlensing event observed, the continuum emitting region of the quasar crossed a caustic at $\geq 72$ percent confidence. This contribution is based on the research presented in Anguita et al. (2008)[1].

The Manchester Microlensing Conference: The 12th International Conference and ANGLES Microlensing Workshop

January 21-25, 2008

Manchester, $U K$

\footnotetext{
* Speaker.
} 


\section{Introduction}

Q2237+0305 is one of the best studied gravitationally lensed quasars. It was discovered by the Center for Astrophysics (CfA) redshift survey [3]. The system shows a barred spiral galaxy at redshift $z_{l}=0.039$, as the main gravitational lens, and four images of a quasar at redshift $z_{s}=1.69$ at a distance of $\sim 1$ " from the center of the lensing galaxy.

Q2237+0305 is the ideal laboratory for quasar microlensing studies. As the quasar images are very close to the core of the lensing galaxy, the microlensing optical depth is high. Additionally, the fact that the quasar is $\sim 10$ times farther away from us than the lensing galaxy makes the projected velocity of the microlenses in the source plane large, thus, having a relatively short timescale of caustic events (i.e. months to years). Moreover, the expected time-delay between the different quasar images is of the order of days [6] and therefore easy to distinguish microlensing from intrinsic variability.

For these reasons this quasar has been monitored for almost 2 decades by different surveys. The most comprehensive set of photometric data for Q2237+0305 is that of the Optical Gravitational Lensing Experiment (OGLE) [15, 10] which shows densely sampled light curves for the four quasar images from 1997 to the present day. We study the 1999 high magnification event seen in image $\mathrm{C}$ of the OGLE light curves using microlensing simulations. For this we have used a flat cosmology with $\Omega_{m}=0.3$ and $H_{0}=70 \mathrm{~km} \mathrm{~s}^{-1} \mathrm{Mpc}^{-1}$.

\section{Data}

In addition to the OGLE dataset ${ }^{1}$ for this event (21/04/99 to 26/12/99), we obtained g' and r'-band data (from 17/05/99 to 08/01/00) of the quasar images, with the $3.5 \mathrm{mts}$ telescope at the Apache Point Observatory (APO).

Using a modified GALFIT [5] version we fitted for the parameters that define the system: The lensing galaxy was parameterized with a de Vaucouleurs profile for the bulge and an exponential profile for the disk, whereas the quasar images where parameterized as point sources. We obtained light curves in both bands for the event of interest (see Fig. 1).

The APO g' light curve is a close match to the OGLE V light curve whereas the APO r' light curve shows a different profile. Therefore when we construct the APO g'-r' color curve for the microlensing event, we can see that it is not flat but shows some variation: in particular, we can observe that there is a blue peak very near the magnification peak.

Chromaticity in a microlensing event has indeed been predicted[12]. From thermal accretion disk models we know that the emission in the central regions is bluer than in the outer regions [9]. So if the caustic size scales are comparable to the accretion disk scales, as in Q2237+0305, then some chromatic microlensing can be observed.

\section{Microlensing Simulations}

In order to compare the observed light curves with simulations, we use source plane magnification patterns created specifically for images $\mathrm{C}$ and $\mathrm{B}$ of the quasar using inverse ray shooting

\footnotetext{
${ }^{1}$ http://bulge.princeton.edu/ ogle/ogle2/huchra.html
} 


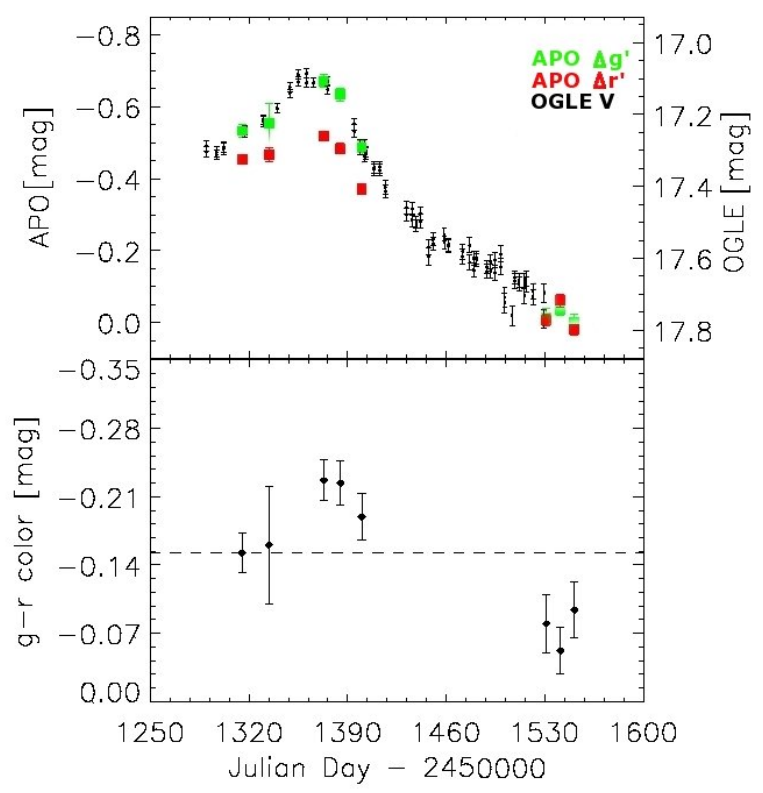

Figure 1: Top panel: OGLE V (black), APO g' (green) and APO r' (red) light curves for the event. Bottom Panel: APO g'-r' color curve. The dashed straight line is shown to guide the eye.

[11]. The parameters required for creating this patterns are the optical depth parameter $(\kappa)$ and the external shear $(\gamma)$ which arise both from the "macro"-lens model of the system. In out case we used the values for $\kappa$ and $\gamma$ obtained by the model presented in Schmidt et al. (1998)[8]. Even though the $\kappa$ parameter can be separated into compactly distributed matter or stars $\left(\kappa_{\star}\right)$ and smoothly distributed matter $\left(\kappa_{c}\right)$ we assumed the fraction $\frac{\kappa_{\star}}{\kappa}$ to be 1.0 as the quasar images are seen within the central kpc of the lensing galaxy.

The quasar magnification depends on the size (or region) of the source that is being magnified. In order to have a sample of the different possibilities of source sizes to be studied, we convolve the raw magnification patterns with different profile sizes. Microlensing fluctuations are relatively insensitive to the source shape, so it is of little consequence the model we choose for the accretion disk [4]. For simplicity have chosen a Gaussian profile for which the extent of the source is defined by the variance $\sigma$. The full width at half maximum (FWHM) is defined as $2.35 \sigma$.

We vary the FWHM of a Gaussian profile from $0.01 E_{R}$ to $0.60 E_{R}$ for quasar image B and from $0.01 E_{R}$ to $1.08 E_{R}$ for quasar image $\mathrm{C}$ (the additional magnification patterns for image $\mathrm{C}$ are used for the color curve fitting explained in the next section).

For a specific convolved pattern we extract light curves, by defining tracks (path of the source in the source plane magnification pattern) with a starting point, direction and velocity. The values are then scaled with the magnification values obtain from the model presented in Schmidt et al. (1998) [8] for each image and converted into magnitudes.

\section{Fitting the Data}

The process of fitting our data is done in two steps: first we only use the densely sampled OGLE light curve, then we add the contraints of the APO color curve. 

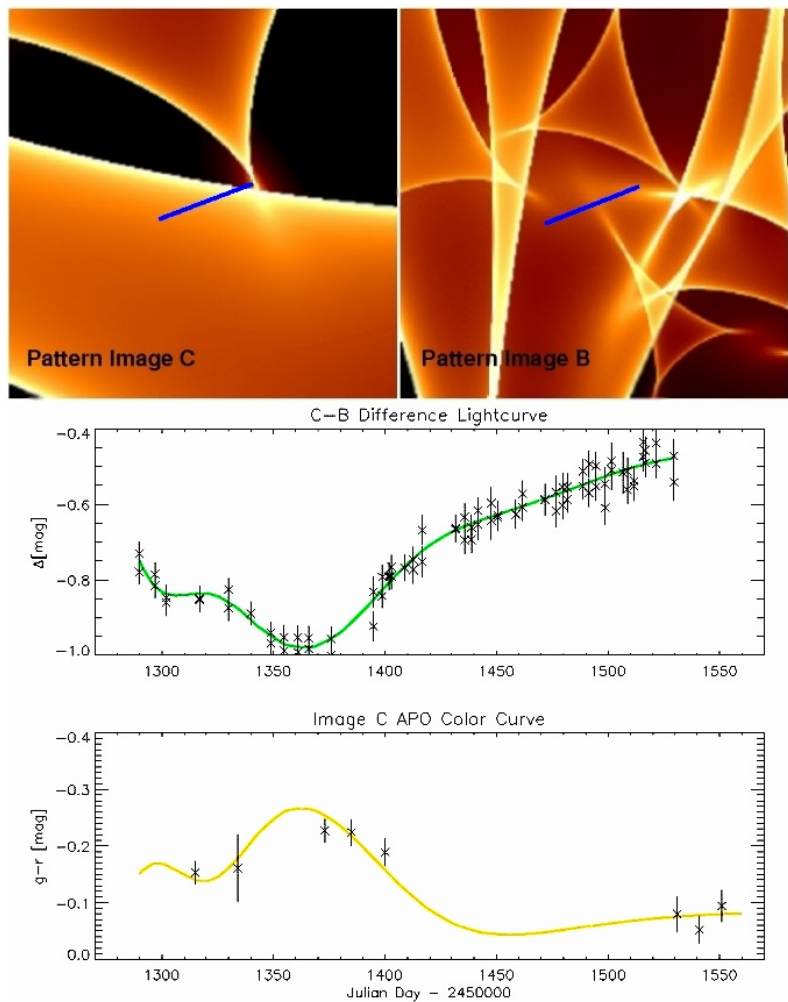

Figure 2: Example track for a $0.05 E_{R}$ (10 pixel) FWHM g'-band source size and a source ratio $\frac{\sigma_{r^{\prime}}}{\sigma_{g^{\prime}}}=1.48$. In the top panels the fitted track (blue) is shown in a $1 E_{R} \times 1 E_{R}$ section of the microlensing patterns. In the middle panel we plot the fitted difference light curve (blue line) and the observed OGLE difference light curve. In the lower panel the best-fitting color curve (yellow line) and the APO g'-r' color curve are shown.

As the quasar might vary intrinsically, we need to account for this variation. For this, and due to the fact that the time delay between the different images is negligible, we use the difference between OGLE light curves for images C and B. Simulated light curves for each image are extracted using tracks over microlensing patterns created independently for each image and convolved with a Gaussian profile with the same size. By repeated light curve extraction with variation of the parameters, the best fit to these are obtained from the comparison with the OGLE C-B difference light curve. What is actually fitted are the parameters that define the track from which both light curves are obtained. This track is constrained to have identical direction and velocity (taking into consideration that the shear direction between images $C$ and $D$ is approximately perpendicular [14]) in both the patterns $\mathrm{C}$ and $\mathrm{B}$, but the starting point can be different among the different patterns.

As the FWHM of each one of the convolved patterns is very small in comparision to the side length of the patterns (of the order of several hundreds) and because it is relatively easy to find good fits due to the huge parameter space that such patterns give, we determine a high number of fits for each one of the convolved patterns. We fit 10,000 tracks with random starting parameters distributed uniformly for patterns convolved with the various source sizes up to $0.60 E_{R}$.

When this minimization is finished we have a track library containing 300,000 tracks with fitted parameters, a source size value and a $\chi^{2}$ attached to each one of them. 
In a second step, we include the chromatic information included in the APO observations. As shown in Fig. 1 the OGLE V light curve and the APO g' light curve are very similar. Thus, we assume that they come from a region of the accretion disk of very similar size. Therefore, the only parameter that defines our dataset that we need to compare to the simulations is the size of the r'-band emitting region of the accretion disk. In particular, as we use the color curve for the event, the parameter of interest is the ratio between the r' and the g' region source sizes.

The parameter allowed to vary is therefore the ratio between the source sizes while the rest remain fixed to the values obtained with the OGLE light curve fitting. In this step we use the full set of convolved patterns created for image $\mathrm{C}$, interpolating between them in order to obtain a continuous distribution for the source ratio parameter. The $\chi^{2}$ obtained due to this fit is used to update the $\chi^{2}$ values of each track in our track library.

\section{Statistics}

Each one of our tracks is a fit to the light and color curves and therefore has a $\chi^{2}$ value attached to it. We infer information from the whole ensemble of models: the track library. Using standard approach for ensemble analysis [7], we assign a likelihood estimator to each track and calculate probability distribution histograms for all the parameters of interest. From this distributions, we can obtain the best-fitting value for each parameter and confidence regions.

There is a degeneracy between the size of the source and the tranverse velocity of it in the microlensing pattern. This is produced because a larger source size smears down steep magnification events, thus making the magnification event appear broader. A slower speed also produces a broader magnification event because it takes more time for the source to cross the magnification region. To cope with this degeneracy we use a prior on the tranverse velocity:

Gil-Merino et al. (2005) (from now on G-M05) found an upper limit of $625 \frac{\mathrm{km}}{\mathrm{s}}$ (90 percent confidence) on the effective transverse velocity of the lensing galaxy in Q2237+0305[2]. We use their probability distribution for the velocity obtained with the largest source size value in a further analysis step where we factor it into our own probability distributions by importance sampling. In other words, we scale the probability of finding a track based on the G-M05 prior, and then reobtain the best-fitting value for each parameter and confidence regions.

\section{Results and Discussion}

As described in the previous section we obtain limits on the parameters of interest from two sets of probability distributions. The first is obtained without using any prior, and the second one includes the G-M05 prior on the transverse velocity of the lensing galaxy. For each probability distribution of a particular parameter we select the 68 percent confidence levels. The distribution, the best-fitting values (or the average of the 68 percent confidence region where no single bestfitting solution is found) and the 68 percent confidence limits for the g' source size and the $\mathrm{r}^{\prime} / \mathrm{g}$ ' source ratio are shown in Fig. 3.

The average Gaussian width we obtain on the OGLE V/APO g' source size without setting the velocity prior is $\sigma_{g^{\prime}}=8.1_{-6.0}^{+5.9} \times 10^{-2} R_{E}$ or $4.6_{-3.4}^{+3.4} \times 10^{15} \sqrt{\frac{M}{0.1 M_{\odot}}} \mathrm{cm}$. When we impose the velocity constraints of G-M05 we obtain an OGLE V/APO g' source size with a Gaussian width of 

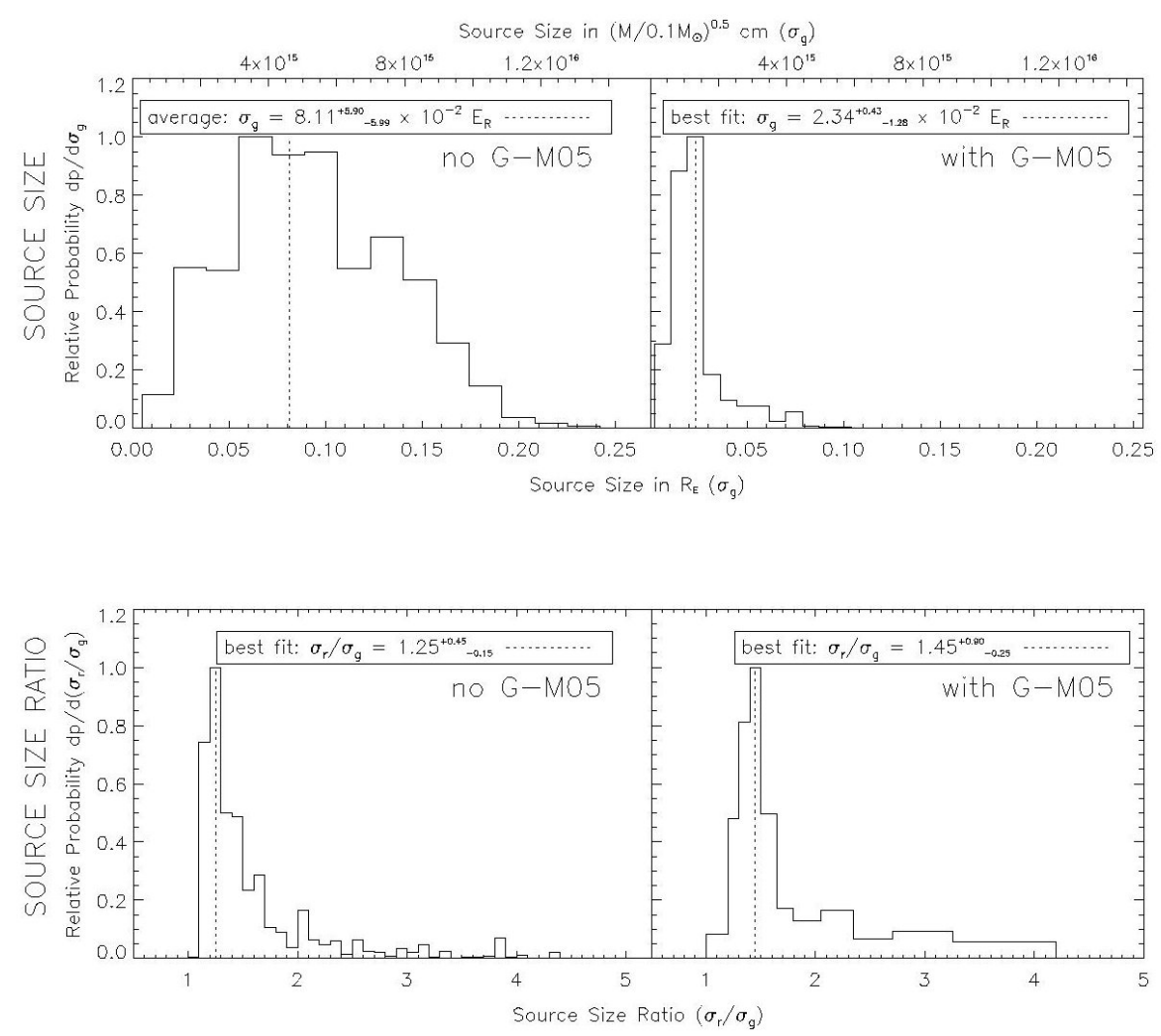

Figure 3: Probability histograms for g' or V source size and r'/g' source size ratio, respectively. The dotted line shows the best-fitting values (or average of the 68 percent confidence region if no single best-fitting value is found). The error bars describe the 68 percent confidence limits.

$\sigma_{g^{\prime}}=2.34_{-1.28}^{+0.43} \times 10^{-2} R_{E}$ which is equivalent to $1.33_{-0.73}^{+0.24} \times 10^{15} \sqrt{\frac{M}{0.1 M_{\odot}}} \mathrm{cm}$. This makes the upper limit tighter by a factor of five compared to the previous result.

The source size ratio between the r'- and g'-band emitting regions of the quasar is $\sigma_{r^{\prime}} / \sigma_{g^{\prime}}=$ $1.25_{-0.15}^{+0.45}$ without, and $\sigma_{r^{\prime}} / \sigma_{g^{\prime}}=1.45_{-0.25}^{+0.90}$ with the G-M05 prior. Both source size ratio distributions are similar and in agreement with what would be expected from a Shakura-Sunyaev [9] thermal model ( 1.5 see Anguita et al 2008 [1]).

By having probabilities attached to each track in our library we can study the nature of the event: whether it was produced by a direct interaccion of the source with a caustic, or indirect magnification by passing outside a cusp. We compute the location of the caustics for each of the magnification patterns using the analytical method from Witt (1990)[13].

For each track we determine whether either the center, the center $\pm \sigma_{g^{\prime}}$ or the center $\pm \sigma_{r^{\prime}}$ of the source touches a caustic at any time. Our analysis shows that tracks that cross a caustic are favored (see Table 1). The source, regardless of the extent, crossed a caustic with a 75 percent and 72 percent of confidence without and with the G-M05 prior, respectively (i.e. the center of the source touched a caustic). 
Table 1: Probabilites of the image $C$ high magnification event being produced by the source crossing a caustic and of the g' and r'-band region touching a caustic. The table shows the obtained values for the probability both using and not using the G-M05 velocity prior.

\begin{tabular}{cccccc}
\hline \multicolumn{3}{c}{ No Prior } & \multicolumn{3}{c}{ With G-M05 Prior } \\
cross & g' & r' & cross & g' & r' \\
\hline 0.75 & 0.92 & 0.97 & 0.72 & 0.89 & 0.94 \\
\hline
\end{tabular}

\section{Summary}

By coupling the OGLE V light curves and APO g' and r' light curves for the high magnification event seen in image C of Q2237+0305 during the year 1999 to source plane microlensing simulations we have been able to obtain limits on the size of regions of the quasar's central engine emitting in these bands: Gaussian width $\sigma_{g^{\prime}}=4.6_{-3.4}^{+3.4} \times 10^{15} \sqrt{M / 0.1 M_{\odot}} \mathrm{cm}$ and $\sigma_{r^{\prime}} / \sigma_{g^{\prime}}=1.25_{-0.15}^{+0.45}$.

Because of the degeneracy between source size and transverse velocity we use a prior on the velocity obtained from the work of Gil-Merino et al. (2005) [2] obtaining: Gaussian width $\sigma_{g^{\prime}}=$ $1.33_{-0.73}^{+0.24} \times 10^{15} \sqrt{M / 0.1 M_{\odot}} \mathrm{cm}$ and $\sigma_{r^{\prime}} / \sigma_{g^{\prime}}=1.45_{-0.25}^{+0.90}$. Both values for the ratio between the source sizes are close to the ratio obtained for a face-on [9] accretion disk (f $\sim 1.5$ ). An independent solution for breaking this degeneracy would be the use of multiband observations with more than one high magnification microlensing event.

Regarding the nature of this event, we show that it was probably produced by the source directly interacting with a caustic as we obtain probabilities of 97 percent and 94 percent that the r'-band emitting region touches a caustic without and with the G-M05 prior, respectively, and of 75 percent and 72 percent that the source center (regardless of size) crossed a caustic without and with the G-M05 prior, respectively.

A comprehensive description of this study is presented in Anguita et al. (2008)[1]

\section{References}

[1] T. Anguita, R. W. Schmidt, E. L. Turner, J. Wambsganss, R. L. Webster, K. A. Loomis, D. Long, R. McMillan, The multiple quasar Q2237+0305 under a microlensing caustic, A\&A 480 (2008), 327-334 [arXiv:0711.4265].

[2] R. Gil-Merino, J. Wambsganss, L. J. Goicoechea, and G. F. Lewis, Limits on the transverse velocity of the lensing galaxy in Q2237+0305 from the lack of strong microlensing variability, A\&A 432 (2005), 83-89 [astro-ph/0411239].

[3] J. Huchra, M. Gorenstein, S. Kent, I. Shapiro, G. Smith, E. Horine, and R. Perley, $2237+0305-A$ new and unusual gravitational lens, AJ 90 (1985), 691-696.

[4] M. J. Mortonson, P. L. Schechter, and J. Wambsganss, Size Is Everything: Universal Features of Quasar Microlensing with Extended Sources, ApJ 628 (2005), 594-603 [astro-ph/ 0408195 ].

[5] C. Y. Peng, L. C. Ho and C. D. Impey, Detailed Structural Decomposition of Galaxy Images, AJ 124 (2002), 266-293 [astro-ph/0204182].

[6] H.-W. Rix, D. P. Schneider, and J. N. Bahcall, Hubble Space Telescope Wide Field Camera imaging of the gravitational lens $2237+0305$, AJ 104 (1992), 959-967. 
[7] M. Sambridge, Geophysical inversion wirh a neighbourhood algorithm - II. Appraising the ensemble, Geophys. J Int. 138 (1999), 727-746.

[8] R. Schmidt, R. L. Webster, and G. F. Lewis, Weighing a galaxy bar in the lens Q2237 + 0305, MNRAS 295 (1998), 488-+ [astro-ph/9708170] .

[9] N. I. Shakura and R. A. Sunyaev, Black holes in binary systems. Observational appearance., A\&A 24 (1973), 337-355.

[10] A. Udalski, M. K. Szymanski, M. Kubiak, G. Pietrzynski, I. Soszynski, K. Zebrun, O. Szewczyk, L. Wyrzykowski, K. Ulaczyk, and T. Wiêckowski, The Optical Gravitational Lensing Experiment. OGLE-III Long Term Monitoring of the Gravitational Lens QSO 2237+0305, Acta Astronomica 56 (2006), 293-305.

[11] J. Wambsganss, Gravitational Lensing: Numerical Simulations with a Hierarchical Tree Code, Journal of Computational and Applied Mathematics 109 (1999), 353-372.

[12] J. Wambsganss and B. Paczynski, Expected color variations of the gravitationally microlensed QSO $2237+0305$, AJ 102 (1991), 864-868.

[13] H. J. Witt, Investigation of high amplification events in light curves of gravitationally lensed quasars, A\&A 236 (1990), 311-322.

[14] H. J. Witt and S. Mao, Interpretation of microlensing events in Q2237 + 0305, ApJ 429 (1994), 66-76.

[15] P. R. Woźniak, A. Udalski, M. Szymański, M. Kubiak, G. Pietrzyński, I. Soszyński, and K. Żebruń, The Optical Gravitational Lensing Experiment: A Hunt for Caustic Crossings in QSO 2237+0305, ApJ 540 (2000), L65-L67 [astro-ph/ 0004287 ]. 